\title{
Multi-Axis Electric Stepper Motor
}

\author{
Vincent Groenhuis ${ }^{1}$, Gijs Rolff ${ }^{2}$, Koen Bosman ${ }^{3}$, Leon Abelmann ${ }^{1,4}$, Stefano Stramigioli ${ }^{1,5}$
}

\begin{abstract}
Multi-axis stepper motors offer a compact solution to actuate multiple degrees of freedom in a mechanical system by a single device. This research presents an electric stepper motor with three coaxial shafts driven by a single stator. The motor dimensions are $42 \times 42 \times 44 \mathrm{~mm}^{3}$ (excluding output shafts), its mass is $0.326 \mathrm{~kg}$ and it includes three rotors of height $7 \mathrm{~mm}$ and different number of teeth.

The maximum torque for one rotor at low speed and $1 \mathrm{~A}$ current is $0.10 \mathrm{~N} \mathrm{~m}$, which is comparable to that of a conventional stepper motor with similar rotor size. When multiple rotors are driven simultaneously, then the total power has to be distributed over the three rotors, resulting in $\mathbf{4 2 \%}$ lower torque on average. The maximum no-load speed is $\mathbf{4 0 0 0}$ revolutions per minute.

The coaxial output shaft configuration poses significant challenges but also unique opportunities in actuating multiple joints of a robotic system. In many applications, the mechanical design can be redesigned to make effective use of the multi-motor design. An example of a three-DOF robot arm is demonstrated that can be efficiently driven by the proposed motor.
\end{abstract}

Index Terms-Actuation and Joint Mechanisms, Engineering for Robotic Systems

\section{INTRODUCTION}

$\mathbf{R}$ OBOTIC systems and many other devices have multiple actuated degrees of freedom (DOFs). Each DOF must be independently driven by some sort of actuating device. Electric motors are most commonly used for this purpose and, by default, each DOF is driven by its own dedicated motor. Examples are stepper motors and brushless DC (BLDC) motors [1], [2]. The number of motors and associated complexities in cabling, electronics and motor mountings then scales linearly with the number of DOFs, which becomes a burden as the device grows in complexity.

It may be advantageous to integrate multiple actuation units into a single space-efficient device, hereby reducing the number of physical actuator entities and associated mounting, cabling and driver electronics requirements.

Of special interest are methods that in some way share certain motor components across multiple outputs, potentially

Manuscript received: February 23, 2021; Revised May 23, 2021; Accepted June 25, 2021.

This paper was recommended for publication by Editor Clement Gosselin upon evaluation of the Associate Editor and Reviewers' comments.

${ }^{1}$ Vincent Groenhuis, Leon Abelmann and Stefano Stramigioli are with Robotics and Mechatronics, University of Twente, $7500 \mathrm{AE}$ Enschede, The Netherlands, e-mail v.groenhuis@utwente.nl, l.abelmann@utwente.nl, s.stramigioli@utwente.nl

${ }^{2}$ Gijs Rolff is with IMS BV, Almelo, The Netherlands, gijs.rolff@multimotor.nl

${ }^{3}$ Koen Bosman is with Eye on Air BV, Enschede, The Netherlands, koen.bosman@multimotor.nl

${ }^{4}$ Leon Abelmann is with KIST Europe, Saarbrücken, Germany.

${ }^{5}$ Stefano Stramigioli is with ITMO University, Saint Petersburg, Russia.

Digital Object Identifier (DOI): see top of this page.

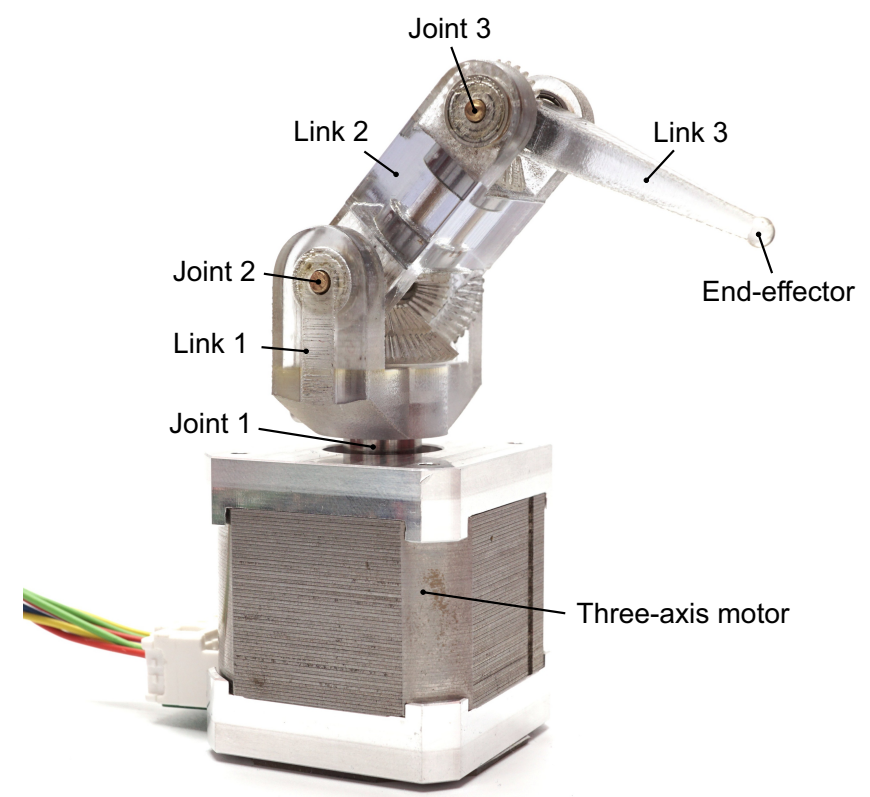

Fig. 1. Our three-axis stepper motor with miniature three-DOF robot arm.

achieving greater space efficiency compared to the aggregation of isolated actuator entities within a single housing.

Earlier research focused on spherical motors in which a ball-shaped rotor fitted with permanent magnets is driven by a set of solenoids in the stator [3], [4], [5], [6], [7], [8], allowing up to three DOFs to be actuated using a single rotor. Without loss of generality, the coils can also be fitted inside a spherical stator, which drives one or two rings of magnets rotating around two or three axes [9], [10]. A critical challenge in the design of spherical motors is how to arrange the magnets and coils in such a way that effective torque can be generated around the relevant axes within the entire workspace.

Motors with coaxially-placed output shafts feature higher flexibility of their driving concepts compared to spherical motors because the individual shafts are mechanically disconnected. Whereas assemblies of two coaxial shafts driven by separate motors are readily commercially available, implementations in which motor components are shared among multiple shafts are much less common. In most studies, the rotors cannot be driven independently and are merely used to generate different shaft velocities without the need for reduction gears [11], [12], [13]. Independent control of multiple coaxial rotors within a single stator has been described in a patent that describes different asynchronous and synchronous rotor types (excluding the stepper motor type) and its combinations [14].

This research describes the development of a multi-axis 


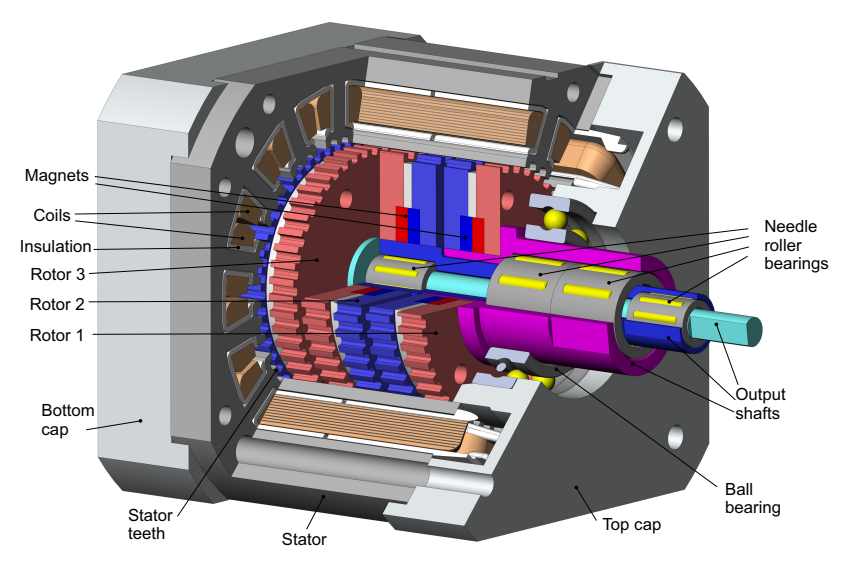

Fig. 2. Cut-out view of the motor showing the rotors, shafts, bearings, stator, coils, insulation and caps. Not visible are the second ball bearing and connector, which are both located inside the bottom cap.

stepper motor with three coaxial output shafts and a singular, homogeneous stator driving all three rotors at once, resulting in a compact system with significant advantages. The specifications are compared to that of a commercially available stepper motor with comparable rotor size. An example application involving a three-link robot arm is demonstrated. Figure 1 shows our three-axis stepper motor with robot arm.

We start with a description of the mechanical design and realization of the proposed three-axis stepper motor in section III. There we also discuss the electronics and the method to construct waveforms driving all three rotors at once, as well as our validation method. The analysis of the new motor is presented in Section III, including torque, maximum no-load speed and back-EMF. We end with a demonstration of the motor on a three degrees of freedom robotic arm.

\section{Methods}

\section{A. Design and construction}

The presented motor consists of the stator with fourteen coils, three rotors mounted on a set of coaxial shafts, two ball bearings, four needle roller bearings, two caps, four fastener screws and one printed circuit board (PCB) with connector. The motor is driven by a controller and driver.

1) Stator: The stator design is based on that of a conventional single-axis stepper motor, with the primary difference that it accommodates additional stator poles and phases. A typical single-axis stepper motor stator includes eight poles arranged in two phases (four poles per phase), whereas an example single-axis BLDC motor stator includes twelve poles arranged in a star configuration of three phases (also four poles each). The proposed design includes fourteen poles arranged in seven phases (two poles per phase). The seven phases are electrically arranged in a star configuration as indicated in Figure 3 and numbered 0 to 6 . Each pole has three teeth spaced $\frac{360^{\circ}}{46}=7.8^{\circ}$ apart to commutate with the rotors properly. The stator is homogeneous, i.e. the teeth configuration is the same for every layer in the stack. The stator height is $27 \mathrm{~mm}$, which is long enough to commutate with all three rotors.

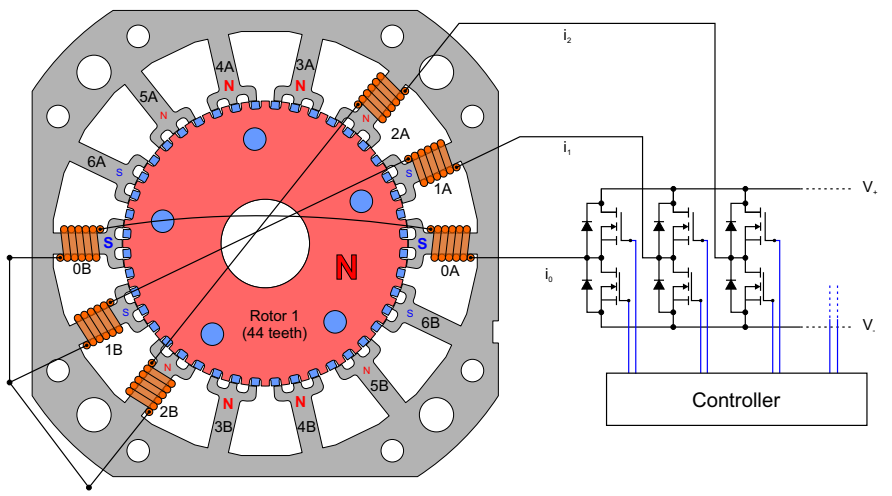

Fig. 3. Coil and electronic configuration of motor, with rotor 1 visible. The stator has fourteen poles with three teeth each, arranged in seven symmetric coil pairs of which three are drawn (0A-0B, 1A-1B and 2A-2B) and connected in a star configuration to the half $\mathrm{H}$-bridge driver. The given magnetization of the seven stator phases attracts rotor 1 towards the shown configuration.

In each coil pair, the two stator poles are radially opposite each other, and the orientation of the coils is such that, when current is applied, both poles have the same magnetization in inward direction (i.e. the induced magnetic fields are radially symmetric).

The stator is constructed from 0.5 -mm-thick electrical steel plates with backlack (voestalpine Stahl GmbH, Linz, Austria). 54 plates are laser-cut with outer dimensions of $42 \times 42 \mathrm{~mm}^{2}$, stacked and aligned with pins, pressed in a compression tool and heated in an oven for stack bonding into a single piece of height $27 \mathrm{~mm}$. Afterwards, the bore is reamed to $\varnothing 25.90 \mathrm{~mm}$.

Insulation parts are 3-D printed from polyethylene terephthalate (PETG) material and inserted into the stator to insulate the fourteen stator poles. Each coil has 50 turns of insulated copper wire with an outside diameter of $0.33 \mathrm{~mm}$. The 28 wire strands are soldered onto the PCB to form seven coil pairs (windings) arranged in a star configuration, resulting in seven leads to the outputs of the seven half H-bridges of the driver.

2) Rotors and shafts: The three rotors each measure $\varnothing 25.80 \times 7 \mathrm{~mm}^{2}$, constructed from two identical halves of height $3 \mathrm{~mm}$ composed of the same electrical steel plates as the stator. A neodymium magnet measuring $\varnothing 15 \times 3 \mathrm{~mm}^{2}$ with a $\varnothing 8 \mathrm{~mm}$ hole (BR Technik Kontor $\mathrm{GmbH}$, Mittelangeln, Germany) is embedded.

The number of teeth $n_{i}$ of the three rotors must conform to the following requirements: $n_{i}$ must be mutually different, divisible by 2 (because coils are arranged in pairs), but not divisible by 7 (otherwise the rotor is uncontrollable), and $n_{i}+$ $n_{j}$ must not be divisible by 7 (otherwise rotors $i$ and $j$ are dependent). Furthermore, $n_{i}$ should be close to 50 to allow comparison with the reference stepper motor, and the spacing between stator poles must be sufficiently wide to allow the stator coils to be wound manually (50 turns). Our choice is $n_{1}=44, n_{2}=46$ and $n_{3}=48$ teeth, respectively, so $n_{r}=$ $42+2 r$ for $r=\{1,2,3\}$. The average rotor teeth count is 46 , and the stator teeth spacing is dimensioned accordingly.

After assembly, some epoxy is applied to the rotor and then machined to the specified diameter. The rotors are press-fitted on their respective stainless-steel shafts. The gap between adjacent rotors is $1 \mathrm{~mm}$. As shown in Figure 2 the orientation 


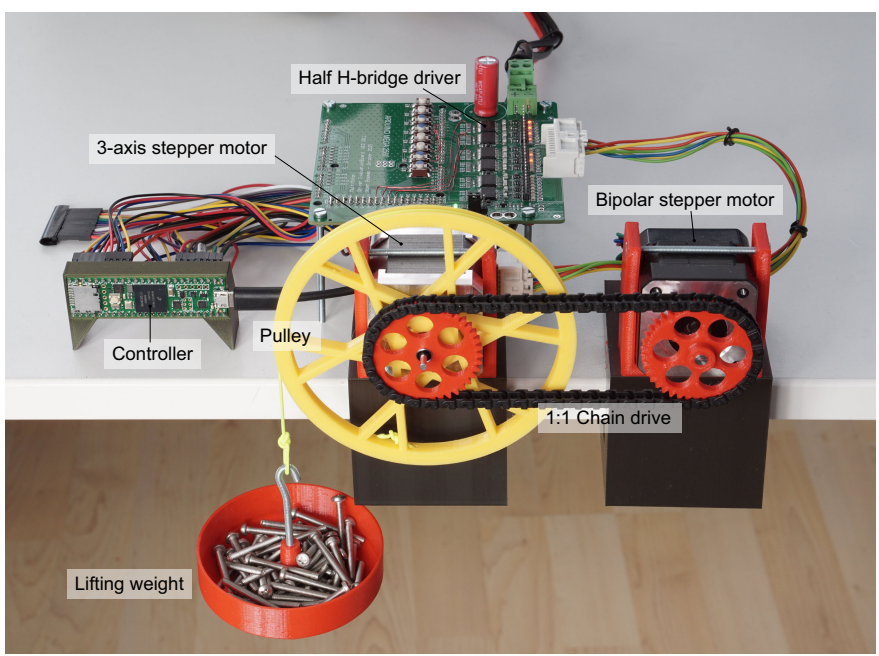

Fig. 4. Measurement setup. The controller calculates the waveforms for the half $\mathrm{H}$-bridge driver, which applies currents to the coils driving the three-axis motor. In torque measurements, a lifting weight with known mass exerts a constant force on a pulley, whereas in back-emf measurements, an external bipolar stepper motor drives one of the three-axis stepper motor shafts over a $1: 1$ chain drive.

of magnets in the rotors are such that adjacent rotors repel each other. Needle roller bearings (type K $3 \times 5 \times 7 \mathrm{TN}$ and $\mathrm{K} 6 \times 9 \times 8 \mathrm{TN})$ are installed between the shafts with tight tolerances to eliminate any possible play, and ball bearings (type 61801) are installed between the outermost shaft and the aluminium caps. The protruding part in the caps match the stator bore for precise alignment, and the stator parts are held together by four screws.

\section{B. Control}

1) Controller and driver: The controller consists of a microprocessing unit of type Teensy 4.1 (PJRC.COM LLC, Sherwood, OR, USA), which houses a NXP i.MX RT1062 processor running an ARM Cortex-M7 core at $600 \mathrm{MHz}$ with hardware floating-point unit (FPU). This microprocessor is fast enough to compute the waveforms using floating-point arithmetic at the same frequency as the hardware pulse-width modulation units for driving the half $\mathrm{H}$-bridges. These are of type TBB6612FNG, which are full H-bridges, one-half of which is used, running at $36.6 \mathrm{kHz}$ and rated at a maximum voltage of $15 \mathrm{~V}$ and $1.2 \mathrm{~A}$ per channel.

2) Coil-rotor commutation: The commutation task is to calculate the coil phase currents $I_{0} . . I_{6}$ such that each rotor $r$ is independently driven to its setpoint angle $\phi_{r}$ by a nominal torque of $T_{r}$.

The coil currents are calculated as

$$
I_{c}=\frac{\sqrt{2}}{K_{\mathrm{T}}} \sum_{r=1}^{3} T_{r} \cos \left(2 \pi c r / 7+\phi_{r}\right)
$$

where $K_{\mathrm{T}}$ is the motor torque constant.

The full mathematical derivation can be found in Appendix A. and a numerical example is provided in Appendix B.

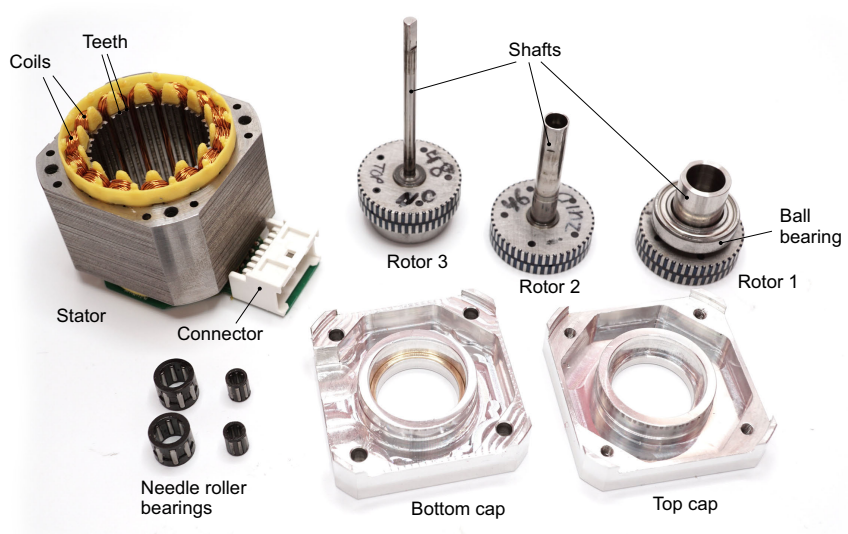

Fig. 5. Components of the three-axis stepper motor.

\section{Validation}

Relevant characteristics of our motor are the low-speed torque, no-load speed and back-EMF voltage, in addition to the consistency of multi-axis position control. The torques, speeds and back-EMF voltages of the different rotors are measured using the setup shown in Figure 4 adapted to the specific measurement.

For comparison, a commercially-available reference bipolar stepper motor with similar rotor dimensions was evaluated under the same conditions (SMNM17S18KG013, 123-3D.nl, Almere, The Netherlands). This reference motor has a total height of $27 \mathrm{~mm}$, rotor height of $9.6 \mathrm{~mm}$ and 50 poles.

The following experiments were conducted:

1) Torque versus current at low speed, single axis: Torque at low speed is evaluated by loading the shaft with known torques, setting the rotor (mechanical) velocity to $0.05 \mathrm{rad} / \mathrm{s}$ and gradually reducing the current in small steps until the rotor starts skipping steps. The load torque is set by hanging weights of $0.025 \mathrm{~kg}$ to $0.200 \mathrm{~kg}$ in steps of $0.025 \mathrm{~kg}$ over a pulley of radius $50 \mathrm{~mm}$. From the resulting current-torque graph, the motor torque constant is derived for each rotor.

The evaluated rotor is not kept at standstill in order to avoid overly optimistic results due to friction between shafts and other irregularities. The stall torque may thus be higher than the measured low-speed torque.

2) No-load speed: The no-load angular speed of each rotor in (both directions) is evaluated at the maximum supply voltage of $15 \mathrm{~V}$ ( $7.5 \mathrm{~V}$ per phase due to the star configuration) by gradually increasing the angular speed until the rotor stalls. The reference bipolar motor is evaluated at $15 \mathrm{~V}$ per phase using sinusoidal waveforms. If necessary, a damping element is added to overcome mid-band instability.

3) Motor velocity constant: The motor velocity constant, which is the inverse of the back-EMF constant, is determined experimentally for each rotor by driving the rotor externally by a secondary stepper motor over a 1:1 chain drive while keeping the other two shafts at standstill. The RMS voltage of one coil phase of the three-axis motor is acquired and scaled by a factor of $\sqrt{2}$ to obtain the approximate peak voltage, which is plotted against the rotational speed of the shaft. The 


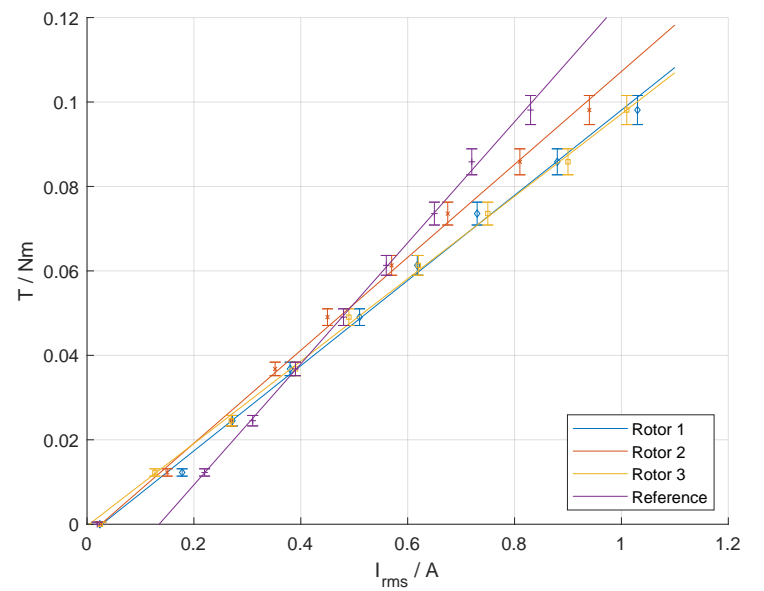

Fig. 6. Torque versus drive current for the three rotors of the three-axis motor. The motor torque constants are comparable to those of the reference motor. Rotor 2 has a $9 \%$ higher torque constant than rotors 1 and 3 .

motor velocity constants are calculated for each rotor from the slopes of the graphs.

4) Multi-axis position control: The correctness of the multiaxis control method is evaluated in a series of tasks. Three dials were constructed to visualize the shaft positions and evaluate the correctness of its motions. In addition, we developed a miniature three-DOF robot arm whose serial kinematic chain consists of three links with a number of bearings, shafts and miter gears. The first link is mounted directly on the motor's first rotor shaft, and series of gears transfer the second and third rotor shafts to the second and third joint, respectively. The spherical tip of the end-effector has a diameter of $4 \mathrm{~mm}$. The forward and inverse kinematics and Jacobian are derived so that the end-effector of the robot arm can move not only in joint space, but also perform movements defined in Cartesian space at constant velocity.

Three different tasks are executed to evaluate the positional consistency, further explained in Section III-D During task execution, the motor power budget is balanced dynamically over the three rotors based on the instantaneous speed and acceleration. The tasks are recorded on video and analyzed for consistency and skipped steps.

\section{RESULTS}

Figure 5 shows the components of our three-axis stepper motor, which is used to actuate the robot arm in Figure 1. The total mass of the motor is $0.326 \mathrm{~kg}$.

\section{A. Torque versus current at low speed}

Figure 6 shows current-torque graphs for all three rotors and the reference motor. The measurement error is approx. $3 \%$ because the slipping threshold is not sharply defined. The motor torque constants $K_{\mathrm{T}}$ and motor size constants $K_{\mathrm{M}}$ are calculated and listed in Table [.

\section{B. Maximum no-load speed}

The observed maximum speeds of the three rotors are shown in Table I. The highest value measured is $4000 \mathrm{rpm}$ for rotor 3.

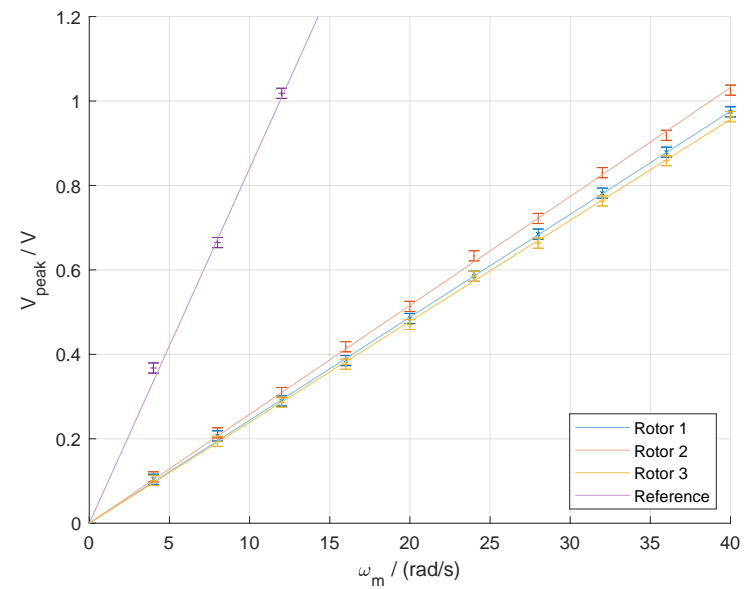

Fig. 7. Back-EMF phase peak voltage versus mechanical angular speed for three rotors compared to the reference motor. The ratio between the corresponding motor velocity constants is approximately 3.4

The reference motor experienced mid-band resonance, which was resolved by gearing it to a secondary stepper motor.

\section{Motor velocity constant}

Figure 7 shows the relationship between the peak voltage of a single coil phase versus the rotation speed for each of the three rotors and the reference motor. The values are included in Table I The average motor velocity constant is $41 \mathrm{rad} \mathrm{s}^{-1} \mathrm{~V}^{-1}$, which is 3.4 times greater than that of the reference motor. The $N / \sqrt{2} K_{\mathrm{V}}$ values (where $N$ denotes the number of phases) are all approximately $0.12 \mathrm{Nm} \mathrm{A}^{-1}$, which is $22 \%$ higher than the respective $K_{\mathrm{T}}$ constants, except for the reference motor, the value of which is $8 \%$ higher than its $K_{\mathrm{T}}$ constant.

\section{Multi-axis position control}

The 3-D printed, three-DOF robot arm developed for the position control evaluation is shown in Figure 1. The mass of the robot arm is $42 \mathrm{~g}$.

For each task, the maximum velocity and acceleration were first tuned such that the task could be performed without skipping steps. The tasks were then recorded and are included in the accompanying video. Our observations per task are as follows:

- Task 1: The three shafts are able to operate at various combinations of speeds, directions and accelerations and return to the starting position. The maximum speed during the movements was $2170 \mathrm{rpm}$ (single axis only) and the maximum acceleration was $5000 \mathrm{rad} / \mathrm{s}^{2}$.

- Task 2: The robot arm is operated in various directions and speeds defined in joint space. The movements can be consistently executed without missing steps when the maximum speed is set to $62 \mathrm{rpm}(6.5 \mathrm{rad} / \mathrm{s})$ and acceleration to $2000 \mathrm{rad} / \mathrm{s}^{2}$.

- Task 3: The end-effector of the robot arm is able to traverse the edges of a cuboid. Some friction is observed between the gears driving joints 2 and 3. From a video analysis, the accuracy and repeatability are estimated to 
TABLE I

MOTOR TORQUE, SIZE AND VELOCITY CONSTANTS AND MAXIMUM NO-LOAD SPEEDS

\begin{tabular}{lcccccc}
\hline \hline Rotor & $\begin{array}{c}\text { Height } \\
\mathrm{mm}\end{array}$ & $\begin{array}{c}K_{\mathrm{T}} \\
\mathrm{N} \mathrm{m} \mathrm{A}^{-1}\end{array}$ & $\begin{array}{c}K_{\mathrm{M}} \\
\mathrm{Nm} / \sqrt{W}\end{array}$ & $\begin{array}{c}K_{\mathrm{V}} \\
\mathrm{rad} \mathrm{V}^{-1} \mathrm{~s}^{-1}\end{array}$ & $\begin{array}{c}N / \sqrt{2} K_{\mathrm{V}} \\
\mathrm{N} \mathrm{m} \mathrm{A}^{-1}\end{array}$ & $\begin{array}{c}\text { No-load speed } \\
\mathrm{RPM}\end{array}$ \\
\hline 1 & 7.0 & 0.097 & 0.0253 & 41.0 & 0.121 & 3600 \\
2 & 7.0 & 0.106 & 0.0276 & 38.8 & 0.128 & 1900 \\
3 & 7.0 & 0.097 & 0.0253 & 41.8 & 0.118 & 4000 \\
Reference & 9.6 & 0.111 & 0.0540 & 11.9 & 0.119 & 1400 \\
\hline
\end{tabular}

be one order of magnitude better than the diameter of the spherical tip, i.e. less than one millimeter.

\section{DISCUSSION}

\section{A. Torque and power}

Rotor 2 can deliver approximately 9\% higher torque than rotors 1 and 3 . This could be attributed to the stator teeth geometry, whose the teeth spacing is optimized for the 46tooth rotor. Additional magnetic effects due to the coaxial arrangement of the rotors may be present, which will require further investigation using a 3D finite-element analysis.

Whereas the motor torque constant $K_{\mathrm{T}}$ is not directly comparable to that of the reference motor due to the different number of turns, the values of the motor size constant $K_{\mathrm{M}}$ shows that the rotors deliver $52 \%$ less torque at the same power than the reference motor. This is attributed partly to the $27 \%$ lower rotor height and partly to the longer wires per turn. It is expected that scaling the motor in width or height will not significantly improve the torque-to-volume ratio, although it might be possible to optimize internal dimensions to achieve higher torques within the same volume.

In practical applications, the total available resistive power of the motor must be shared among the three rotors. With a motor constant of $0.026 \mathrm{Nm} / \sqrt{W}$ and a momentary power of $20 \mathrm{~W}$, one could perform load-dependent power balancing, e.g. dedicate $50 \%$ of the total power to rotor $1,25 \%$ to rotor 2 and $25 \%$ to rotor 3 , resulting in $0.0253 \cdot \sqrt{10} \mathrm{~N} \mathrm{~m}=0.080 \mathrm{~N} \mathrm{~m}$ of torque in rotor $1,0.062 \mathrm{Nm}$ in rotor 2 and $0.057 \mathrm{~N} \mathrm{~m}$ in rotor 3. The combined torque is $0.20 \mathrm{~N} \mathrm{~m}$, or $0.045 \mathrm{Nm} / \sqrt{W}$, which is $8 \%$ less than that of the reference motor $(0.054 \mathrm{Nm} / \sqrt{W})$.

\section{B. Maximum no-load speed and motor torque constant}

The highest observed no-load speed of $4000 \mathrm{rpm}$ is significantly higher than that of the reference motor. The limiting factor is the back-EMF voltage: With a motor velocity constant $K_{\mathrm{V}}=390 \mathrm{rpm} / \mathrm{V}$, the peak back-EMF voltage is $10 \mathrm{~V}$ or 7.1 V RMS. Although the driver is technically unable to deliver peak voltages of $10 \mathrm{~V}$ per phase, it is able to overcome the back-EMF at the given speed.

The supply voltage is limited by the specific H-bridges used in this setup and could be increased by using different chips (e.g. DRV8844). It is also possible to use full H-bridges (as employed in driving the reference motor) instead of a star configuration to enable the full supply voltage to be used at each coil phase at the cost of doubling the number of wires.

\section{Multi-axis position control}

The task with the small dials could be executed at significantly higher speeds and accelerations than the robot arm tasks, which can be attributed to the relatively low inertia of the dials. The tasks involving the robot arm shows reasonable speed and acceleration for a system of its size, and it could be further optimized using model-based dynamic acceleration control based on the actual position of the links, which has a significant effect on the rotor inertia.

\section{General discussion}

The presented three-axis motor shows that it is possible to actuate multiple rotors with a single set of coils shared among all rotors. This has a number of advantages:

- Compact construction: Multiple rotors can be stacked without significant gaps between them.

- High phase count: Each rotor is driven by seven phases, whereas bipolar stepper motors use only two phases. The torque is therefore more constant, the cogging torque and ripple are significantly reduced and theoretical stepping accuracy is significantly improved compared to bipolar stepper motors.

- Dynamic power balancing: The power budget can be dynamically distributed over the rotors based on the instantaneous load, speed and acceleration demands per rotor.

- Low wire count: The three rotors are driven by a total of seven wires in a star configuration, requiring only seven half $\mathrm{H}$-bridges in the driver.

- Easy integration: The motor can be directly installed in multi-DOF robotic systems with specific kinematic configurations.

However, there are also disadvantages that must be investigated further to quantify their effects:

- Waveform superposition: The total driving waveform is a superposition of three individual waveforms, one for each rotor. Whereas the waveform driving a specific rotor should not affect the other two rotors, it may do so due to nonlinear effects, which also results in increased resistive power losses. The three-axis motor is therefore inherently less efficient than three individual motors, and the three rotors cannot simultaneously provide maximum torque.

- Fabrication of coaxial shafts: It is difficult to manufacture shafts with needle roller bearings because these have to be precisely machined to accommodate the bearings without 
clearance. This issue might be mitigated in future designs by using miniature ball bearings.

- Mechanical transfer to individual joints: In many kinematic configurations, it is disadvantageous to have the three shafts positioned coaxially because it complicates the mechanical transfer to the respective joints.

\section{CONCLUSION}

A compact three-axis electric stepper motor has been developed with a single stator and three rotors. The average torque constant is $0.10 \mathrm{~N} \mathrm{~m} \mathrm{~A}^{-1}$, and the motor velocity constant is $41 \mathrm{rad} \mathrm{s}^{-1} \mathrm{~V}^{-1}$. The motor constant is $0.026 \mathrm{Nm} / \sqrt{W}$, which is $52 \%$ smaller than that of a reference motor with a $37 \%$ higher rotor. Therefore, the three-axis motor presented here has a higher resistive power dissipation at the same torque.

The highest measured speed is $4000 \mathrm{rpm}$, which is 2.9 times faster than that of the reference motor. This maximum speed is limited by the motor velocity constant in combination with the $15 \mathrm{~V}$ supply voltage and the stator windings arranged in star topology.

The controller is able to drive each rotor independently to its setpoint position at the desired speed. The demonstrated three-DOF robot arm is able to perform tasks defined in joint space and Cartesian space with maximum velocities of $62 \mathrm{rpm}$ $\left(6.5 \mathrm{rad} \mathrm{s}^{-1}\right)$ and maximum acceleration of $2000 \mathrm{rad} / \mathrm{s}^{2}$.

Our motor has shown good potential to serve as an actuator of robotic systems with suitable kinematic configurations. In comparison to a solution with individual motors, the example robot arm is relatively easy to develop, thanks to the coaxial output shafts. The motor is able to balance the power dynamically over the different joints based on the instantaneous torque demands, whereas the seven-wire connection simplifies the cable bundle to the driver electronics. However, the maximum rated torque cannot be delivered simultaneously to all shafts, and the concentric arrangement may be too restrictive in kinematic designs with unfavorable joint configurations. Although the merits of our three-axis motor are heavily applicationdependent, it is safe to say there are various applications in which traditional approaches using single-shaft stepper motors can be effectively exchanged for implementations with the proposed electric multi-axis stepper motor.

\section{APPENDIX}

\section{A. Mathematical background for coil-rotor commutation}

The electrical angle $\phi_{r}$ of physical rotor $r$ is defined as the mechanical angle $\alpha_{r}$ multiplied by the pole count $n_{r}$, i.e. $\phi_{r}=$ $n_{r} \alpha_{r}$. The electrical phase is the electrical angle modulus $2 \pi$.

The stator poles are spaced $2 \pi / 14 \mathrm{rad}$ apart. A phase $\beta_{c r}$ is associated with each combination of coil $c \in[0 . .6]$ and rotor $r \in[0 . .6]$, which is computed as follows:

$$
\begin{aligned}
\beta_{c r} & =\frac{2 \pi c n_{r}}{14} \quad(\bmod 2 \pi) \\
& =\frac{2 \pi c(42+2 r)}{14} \equiv \frac{2 \pi c r}{7} \quad(\bmod 2 \pi) .
\end{aligned}
$$

The interpretation of $\beta_{c r}$ is that, when a positive current $I_{c}$ is applied to coil $c$, a magnetic field is created, which attracts and/or repels the (north/south) poles of rotor $r$ such that the rotor is pulled towards the electrical phase $\beta_{c r}$.

For simplicity, we assume that the coil-rotor torque is a pure sinusoidal function of the electrical angle $\phi$. In reality, there may be higher-frequency harmonics and/or some dependency on angular velocity $\dot{\phi}$ due to physical charactistics such as the exact geometry of the teeth, reluctance and saturation effects, eddy currents etc. However, considering the high number of phases, it is reasonable to use a sinusoidal approximation.

We define the following coil-rotor "action phasor" $A_{c r}$ to aid in the calculations:

$$
A_{c r}=\frac{\sqrt{2} K_{\mathrm{T}}}{7} I_{c} e^{-i \beta_{c r}},
$$

where $K_{\mathrm{T}}$ is the motor torque constant in $\mathrm{N} \mathrm{m} \mathrm{A}^{-1}$. The $\sqrt{2} / 7$ factor is needed to account for the number of coils and the complex exponential factor.

The total action phasor of all seven coils on rotor $r$ is computed as follows:

$$
\begin{aligned}
A_{r} & =\sum_{c=0}^{6} A_{c r}=\sum_{c=0}^{6} \frac{\sqrt{2} K_{\mathrm{T}}}{7} I_{c} e^{-i \beta_{c r}} \\
& =\frac{\sqrt{2} K_{\mathrm{T}}}{7} \sum_{c=0}^{6} I_{c} e^{-i 2 \pi c r / 7} .
\end{aligned}
$$

This can be reformulated using the discrete Fourier transform $\mathcal{F}(\vec{I})$ in which the coil current vector $\vec{I}$ contain the samples and $\vec{A}$ the complex frequencies:

$$
\vec{A}=\frac{\sqrt{2} K_{\mathrm{T}}}{7} \mathcal{F}(\vec{I})
$$

We have $A_{0}=0$ because $\sum_{c=0}^{6} I_{c}=0$, and $A_{7-r}=A_{r}^{*}$. Therefore, vector $\vec{A}$ is fully defined by $\left(A_{1}, A_{2}, A_{3}\right)$.

The phase of phasor $A_{r}(r=1 . .3)$ defines the electrical phase to which rotor $r$ is being pulled. The magnitudes of $A_{r}$ and $A_{7-r}$ are related to the amplitude of the magnetic energy, i.e. half the difference between maximum and minimum energy when the rotor is being rotated.

If we define the magnetic energy for rotors $r=\{1,2,3\}$ as

$$
V_{r}(\phi)=-\frac{1}{2}\left(A_{r} e^{-i \phi}+A_{r}^{*} e^{i \phi}\right)=-\operatorname{Re}\left(A_{r} e^{-i \phi}\right),
$$

then the torque on rotor $r$ follows from the gradient of this magnetic energy:

$$
\begin{aligned}
T_{r}(\phi) & =-\nabla V_{r}(\phi) \\
& =-\frac{d}{d \phi}\left(-\frac{1}{2}\left(A_{r} e^{-i \phi}+A_{r}^{*} e^{i \phi}\right)\right) \\
& =\frac{1}{2}\left(-i A_{r} e^{-i \phi}+i A_{r}^{*} e^{i \phi}\right) \\
& =\operatorname{Im}\left(A_{r} e^{-i \phi}\right)
\end{aligned}
$$

The maximum absolute torque $\max \left|T_{r}(\phi)\right|$ is reached when $\phi$ leads or lags $\angle A_{r}$ by $\frac{\pi}{2}$. In other words,

$$
T_{r}\left(\angle A_{r} \pm \frac{\pi}{2}\right)= \pm\left|A_{r}\right| \text {. }
$$


Driving the rotor can be regarded as "riding the wave", where $V_{r}(\phi)$ defines the wave and $\phi$ the particle on the wave. The wave amplitude must be high enough that the particle does not jump over the top, which would correspond to skipping steps.

The action phasor vector $\vec{A}=\left(A_{1}, A_{2}, A_{3}\right)$ is typically a time-varying function that can be fully determined by the application. At constant electrical angular speed $\omega_{e}$ and nominal torque $T_{0}$, it can be represented as $A_{r}=T_{0} e^{i \omega_{e} t}$. A numerical example is given in Appendix B

The reference motor is a bipolar stepper motor with two phases. In micro-stepping mode, its phase currents $I_{0}, I_{1}$ are computed as

$$
\left(I_{0}, I_{1}\right)=\left(\operatorname{Re}\left(A / K_{\mathrm{T}}\right), \operatorname{Im}\left(A / K_{\mathrm{T}}\right)\right) .
$$

For the presented three-axis motor with shared coils, we instead inverse Equation 7 by calculating the inverse discrete Fourier transformation to compute the coil currents

$$
\begin{aligned}
\vec{I} & =\frac{7}{\sqrt{2} K_{\mathrm{T}}} \mathcal{F}^{-1}(\vec{A}) \\
I_{c} & =\frac{7}{\sqrt{2} K_{\mathrm{T}}} \cdot \frac{1}{7} \sum_{r=0}^{6} A_{r} e^{i 2 \pi c r / 7} \\
& =\frac{1}{\sqrt{2} K_{\mathrm{T}}} \sum_{r=1}^{3} A_{r} e^{i 2 \pi c r / 7}+\left(A_{r} e^{i 2 \pi c(7-r) / 7}\right)^{*} \\
& =\frac{\sqrt{2}}{K_{\mathrm{T}}} \operatorname{Re}\left(\sum_{r=1}^{3} A_{r} e^{i 2 \pi c r / 7}\right) \\
& =\frac{\sqrt{2}}{K_{\mathrm{T}}} \sum_{r=1}^{3}\left|A_{r}\right| \cos \left(2 \pi c r / 7+\angle A_{r}\right) .
\end{aligned}
$$

Equation 19 (consistent with Equation 1) is a real-valued function used by the controller to compute the current vector $\vec{I}=\left(I_{0}, I_{1}, I_{2}, I_{3}, I_{4}, I_{5}, I_{6}\right)$ to drive through the seven coil phases.

The motor has seven coils phases arranged in a star configuration. Kirchhoff's current law states that the sum of the currents through these coils must be 0 . Indeed it is:

$$
\begin{aligned}
\sum_{c=0}^{6} I_{c} & =\sum_{c=0}^{6} \frac{1}{\sqrt{2} K_{\mathrm{T}}} \sum_{r=0}^{6} A_{r} e^{i 2 \pi c r / 7} \\
& =\frac{1}{\sqrt{2} K_{\mathrm{T}}} \sum_{r=0}^{6} A_{r} \sum_{c=0}^{6} e^{i 2 \pi c r / 7}=0 .
\end{aligned}
$$

Therefore, there are six independent input variables in the physical system of the motor. This matches the number of independent variables in the $\vec{A}$ phasor vector, which makes sense to achieve a fully controllable system with a minimum of coil phases.

An example physical interpretation is as follows: The coilrotor commutation values form circular waves with different periods (called wave numbers, harmonics or wavelengths wrapped around $2 \pi$ ) orthogonal to each other. The efforts sent through the coils is a composition of waves with these three different periods, where each wave is picked up by the rotor matching its wavelength.

The RMS current is calculated with help of Parseval's (or Plancherel's) theorem, which states that

$$
\sum_{c=0}^{6}\left|I_{c}\right|^{2}=\frac{1}{7} \sum_{r=0}^{6}\left|\frac{7}{\sqrt{2} K_{\mathrm{T}}} A_{r}\right|^{2}=\frac{7}{2 K_{\mathrm{T}}^{2}} \sum_{r=0}^{6}\left|A_{r}\right|^{2} .
$$

Therefore,

$$
I_{\mathrm{rms}}^{2}=\frac{1}{7} \sum_{c=0}^{6} I_{c}^{2}=\frac{1}{2 K_{\mathrm{T}}^{2}} \sum_{r=0}^{6}\left|A_{r}\right|^{2}
$$

Taking the square root yields

$$
I_{\mathrm{rms}}=\frac{1}{\sqrt{2} K_{\mathrm{T}}} \sqrt{\sum_{r=0}^{6}\left|A_{r}\right|^{2}} .
$$

When only one rotor $r=1$ is considered $\left(A_{q}=0\right.$ for $q \notin\{1,6\})$, this simplifies to

$$
\begin{aligned}
I_{\mathrm{rms}, 1} & =\frac{1}{\sqrt{2} K_{\mathrm{T}}} \sqrt{\left|A_{1}\right|^{2}+\left|A_{6}\right|^{2}} \\
& =\frac{1}{\sqrt{2} K_{\mathrm{T}}} \sqrt{2\left|A_{1}\right|^{2}}=\frac{1}{K_{\mathrm{T}}}\left|A_{1}\right|,
\end{aligned}
$$

which is consistent with the definition of the torque constant $K_{\mathrm{T}}$ and Equation 13 .

There exists a direct relation between the motor torque constant $K_{\mathrm{T}}$ (in $\mathrm{N} \mathrm{m} \mathrm{A}^{-1}$ ) and the motor velocity constant $K_{\mathrm{V}}$ (in $\operatorname{rad~s}^{-1} \mathrm{~V}^{-1}$ using peak voltage). We use the power balance to derive this relation:

$$
P=N V_{\mathrm{rms}} I_{\mathrm{rms}}=T \dot{\alpha},
$$

where $N$ denotes the number of phases, i.e. $N=7$ for the three-axis motor and $N=2$ for the reference motor.

We have $V_{\text {peak }}=\sqrt{2} V_{\text {rms }}, K_{\mathrm{T}}=T / I_{\text {rms }}$ and $K_{\mathrm{V}}=\dot{\alpha} / V_{\text {peak }}$, which leads to

$$
\frac{N}{\sqrt{2}} V_{\text {peak }} \frac{T}{K_{\mathrm{T}}}=T K_{\mathrm{V}} V_{\text {peak }} .
$$

Therefore,

$$
K_{\mathrm{T}}=\frac{N}{\sqrt{2} K_{\mathrm{V}}} .
$$

\section{B. Numerical example}

We use $K_{\mathrm{T}}=0.10 \mathrm{Nm} \mathrm{A}^{-1}$ and $R=2.1 \Omega$ per phase.

Suppose that rotor 1 is held stationary with nominal torque $0.05 \mathrm{~N} \mathrm{~m}$, rotor 2 is moving at an electrical angular speed of $1 \mathrm{rad} / \mathrm{s}$ with nominal torque $0.1 \mathrm{Nm}$ and rotor 3 is oscillating with a frequency of $2 \mathrm{rad} / \mathrm{s}$ at amplitude $\pi \mathrm{rad}$ and nominal torque $0.15 \mathrm{Nm}$. The action phasor vector then becomes

$$
\overrightarrow{A(t)}=\left(0.05,0.1 e^{i t}, 0.15 e^{i \pi \cos 2 t}\right) .
$$


At $t=1$, this evaluates to

$$
\overrightarrow{A(1)}=(0.050,0.054+0.084 i, 0.039-0.145 i) \text {. }
$$

The coil currents at $t=1$ are computed using Equation 19 .

$$
\vec{I}=(2.02,0.04,0.58,-3.21,2.64,-1.59,-0.50) .
$$

The sum is 0 (up to rounding error). The RMS current is 1.87 A, which is consistent with Equation 24

Applying the discrete Fourier transform to Equation 32 yields

$$
\begin{array}{r}
\vec{f}=(0,2.47,2.67+4.17 i, 1.93-7.17 i, 1.93+7.17 i \\
2.67-4.17 i, 2.47)
\end{array}
$$

Multiplying the values by $\sqrt{2} K_{\mathrm{T}} / 7$ yields

$$
\begin{aligned}
& \frac{\sqrt{2} \cdot 0.1}{7} \vec{f}=\overrightarrow{A^{\prime}(1)}=(0,0.05,0.054-0.084 i \\
& 0.039+0.145 i, 0.039-0.145 i, 0.054+0.084 i, 0.050)
\end{aligned}
$$

This is consistent with the values of $\vec{A}$ in Equation 31 because $\vec{A}$ includes only the three independent values of the sevencomponent vector $\overrightarrow{A^{\prime}}$.

Using a phase resistance of $\mathrm{R}=2.1 \Omega$, we find that the total resistive power dissipation in the three-axis motor is

$$
P=7 I_{\text {rms }}^{2} R=51.4 \mathrm{~W} .
$$

The resistive power $P$ can also be decomposed per rotor, based on the power spectrum

$$
\begin{aligned}
& P_{r}=7\left|\frac{1}{\sqrt{2} K_{\mathrm{T}}} A_{r}\right|^{2} R \\
& \vec{P}=(0,1.8,7.4,16.5,16.5,7.4,1.8) \mathrm{W} .
\end{aligned}
$$

The power in rotor $r$ equals $P_{r}+P_{7-r}$, so the total power in rotors 1,2 and 3 is $3.7 \mathrm{~W}, 14.7 \mathrm{~W}$ and $33.1 \mathrm{~W}$, respectively. The sum indeed equals $51.4 \mathrm{~W}$ (up to rounding error).

The rotor torques can be computed using these values and the motor size constants $K_{\mathrm{M}}$ as follows:

$$
\begin{aligned}
T_{r} & =K_{\mathrm{M}}\left(P_{r}+P_{7-r}\right)^{2} \\
T_{1 . .3} & =(0.050,0.100,0.150) \mathrm{Nm} .
\end{aligned}
$$

These values are consistent with the nominal torque values in Equation 31. Actually, the motor may not be able to deliver the requested torque due to nonlinear effects. Nevertheless, the numeric example demonstrates that, although the coils are shared among several rotors, it is still possible to control the torque and phase of each rotor independently.

\section{ACKNOWLEDGMENTS}

The authors would like to sincerely thank Pieter Post and André Eppingbroek for producing the mechanical parts, Jeroen Houwers for technical assistance, Jan Bongaarts for winding the coils, Sander Smits for 3-D printing parts of the robot arm, voestalpine Stahl GbmH for providing electrical steel plates, Lilli-Marie Pavka for proofreading and Johan Paulides plus the anonymous reviewers for the valuable feedback on the initial manuscript.

\section{REFERENCES}

[1] A. Hughes and B. Drury, Electric Motors and Drives: Fundamentals, Types and Applications. Elsevier, 2019.

[2] J. He and F. Gao, "Mechanism, Actuation, Perception, and Control of Highly Dynamic Multilegged Robots: A Review," Chinese Journal of Mechanical Engineering (English Edition), vol. 33, no. 1, 2020. [Online]. Available: https://doi.org/10.1186/s10033-020-00485-9

[3] G. S. Chirikjian and D. Stein, "Kinematic design and commutation of a spherical stepper motor," IEEE/ASME Transactions on Mechatronics, vol. 4, no. 4, pp. 342-353, 1999.

[4] K. Kaneko, I. Yamada, and K. Itao, "A spherical dc servo motor with three degrees of freedom," Journal of Dynamic Systems, Measurement and Control, Transactions of the ASME, vol. 111, no. 3, pp. 398-402, 1989.

[5] C. Xia, H. Li, and T. Shi, "3-D magnetic field and torque analysis of a novel halbach array permanent-magnet spherical motor," IEEE Transactions on Magnetics, vol. 44, no. 8, pp. 2016-2020, 2008.

[6] Y. Chen, I. Godage, H. Su, A. Song, and H. Yu, "Stereotactic Systems for MRI-Guided Neurosurgeries: A State-of-the-Art Review," Annals of Biomedical Engineering, vol. 47, no. 2, pp. 335-353, 2019.

[7] B. Dehez, G. Galary, D. Grenier, and B. Raucent, "Development of a spherical induction motor with two degrees of freedom," IEEE Transactions on Magnetics, vol. 42, no. 8, pp. 2077-2089, 2006.

[8] S. Maeda, K. Hirata, and N. Niguchi, "Dynamic analysis of an independently controllable electromagnetic spherical actuator," IEEE Transactions on Magnetics, vol. 49, no. 5, pp. 2263-2266, 2013.

[9] M. David, "( 12 ) Patent Application Publication ( 10 ) Pub . No .: US 2017 / 0215756A1," pp. 2015-2018, 2017.

[10] Y. Sakaidani, K. Hirata, S. Maeda, and N. Niguchi, "Feedback control of the 2-DOF actuator specialized for 2-axes rotation," IEEE Transactions on Magnetics, vol. 49, no. 5, pp. 2245-2248, 2013.

[11] G. Liu, G. Qiu, J. Shi, and F. Zhang, "Study on Counter-Rotating DualRotor Permanent Magnet Motor for Underwater Vehicle Propulsion," IEEE Transactions on Applied Superconductivity, vol. 28, no. 3, 2018.

[12] M. Fukuoka, K. Nakamura, H. Kato, and O. Ichinokura, "A novel flux-modulated type dual-axis motor for hybrid electric vehicles," IEEE Transactions on Magnetics, vol. 50, no. 11, pp. 1-4, 2014.

[13] M. Thoms, "Synchronous Permanent Magnet Machine, Patent US 2009 / 0309443 A1," 2009.

[14] A. Patzak and F. Bachheibl, "Patent Application Publication: US 2019 / 0252929 A1," 2019. 